\title{
Psychosocial challenges in palliative care: Bridging the gaps using digital health
}

\author{
Abida Sultana*, Samia Tasnim, Rachit Sharma, Priyanka Pawar, \\ Sudip Bhattacharya, Md Mahbub Hossain
}

\begin{abstract}
Psychosocial health is a major palliative care concern globally. Patients and caregivers engaged in palliative care may experience deteriorated mental and social health conditions. Global evidence informs a high burden of depression, anxiety, psychological distress, and other mental health problems among the palliative care populations. Those challenges aggravate the preexisting palliative care issues, such as lack of access and suboptimal quality of care. Palliative caregiving, both in the institutional and community settings, should be strengthened, incorporating psychosocial support for the patients and their caregivers. The recent advancements in digital health technologies have shown promising impacts in facilitating health communication, decisionmaking, and delivering psychosocial interventions using digital devices and platforms. Adopting evidence-based interventions can help the users to access mental health resources and communicate with each other to promote shared decision-making and management of health problems. Healthcare organizations and systems may explore opportunities to advance psychosocial care using digital technologies. Leveraging advanced technologies in palliative caregiving may require multifaceted research exploring potential barriers and opportunities at the user, institutional, and systems levels. Nonetheless, palliative care policies and health systems measures should be strengthened to develop and implement digital support systems that may improve psychosocial health and overall palliative care outcomes.
\end{abstract}

Keywords: Palliative care; hospice care; end of life care; telemedicine; digital health

\footnotetext{
* Correspondence: Dr. Abida Sultana, Gazi Medical College, Khulna 09000, Bangladesh. Email: a.sultana21@ outlook.com
} 


\section{Introduction}

Palliative care has critical psychosocial impacts on the patients and their informal caregivers. ${ }^{[1,2]}$ The magnitude of such problems can be examined from different perspectives. First, the patients receiving palliative care may have poor mental health statuses due to their primary disease as well as persistent psychological stressors in their altered lifestyle. ${ }^{[1,3]}$ Second, access to mental health services remains a major challenge globally. ${ }^{[4-7]}$ For this reason, people under palliative care who has clinically significant mental health issues may not receive the care they need. Third, caring for someone with palliative conditions can be burdensome, especially if the caregiver is also experiencing socioeconomic challenges resulting in a cluster of psychological stressors for them. ${ }^{[1-}$

${ }^{3,8]}$ Fourth, palliative care is neither available nor accessible to a major proportion of the global population. ${ }^{[9]}$ A lack of care keeps affecting the health and quality of living among the affected individuals and their caregivers, which subsequently impacts their psychosocial wellbeing. ${ }^{[10]}$ Nonetheless, people who serve as palliative care providers often spend long hours, stressful worklife, and limited mental health resources. ${ }^{[11]}$ Therefore, critical issues such as emotional burnout, sleep deprivation, depression, and other mental health problems appear to be highly prevalent in palliative care providers. ${ }^{[1,12]}$ It is essential to examine and address such psychosocial challenges that are impacting primary stakeholders in palliative care, including the patients, family caregivers, and healthcare providers.

Several studies have shown a high burden of psychological problems among palliative care patients. A study assessed psychological health conditions among 381 cancer patients who were receiving palliative care and found the prevalence rates of anxiety and depression were $13.9 \%$ and $20.7 \% \cdot{ }^{[13]}$ Another study from Australia reported a dual burden of anxiety and depression among about $25 \%$ of the 266 palliative care patients. ${ }^{[14]}$ Similar studies from multiple global settings have 
shown a high prevalence of psychosocial challenges in patients receiving palliative care. A metaanalytic review of 24 studies with 4007 individuals from seven countries found that the pooled prevalence of depression, major depression, and minor depression were $16.5 \%$ (95\% confidence interval $[\mathrm{CI}] 13 \cdot 1-20 \cdot 3), 14 \cdot 3 \%(95 \% \mathrm{CI}: 11 \cdot 1-17 \cdot 9)$, and $9 \cdot 6 \%(3 \cdot 6-18 \cdot 1)$, respectively. ${ }^{[15]}$ Moreover, the prevalence rates of adjustment disorder and anxiety disorders were $15 \cdot 4 \%(10 \cdot 1-$ $21 \cdot 6)$ and $9 \cdot 8 \%(6 \cdot 8-13 \cdot 2)$, respectively. Furthermore, the synthesis of 70 studies with 10071 individuals from 14 countries in haemato-oncological settings reported the prevalence of depression, anxiety, adjustment disorders, dysthymia, and any mood disorders were $16 \cdot 3 \%$ (95\% CI: $13 \cdot 4-19 \cdot 5), 10 \cdot 3 \%(95 \%$ CI: $5 \cdot 1-17 \cdot 0), 19 \cdot 4 \%(95 \%$ CI: $14 \cdot 5-24 \cdot 8), 2 \cdot 7 \%$ (95\% CI: $1 \cdot 7-$ $4 \cdot 0)$, and $38 \cdot 2 \%(95 \%$ CI: $28 \cdot 4-48 \cdot 6) .{ }^{[15]}$ Such psychological challenges are also common among informal caregivers of patients receiving palliative care services. A study found that $62.7 \%$ of informal caregivers to palliative care patients had symptoms of depression. ${ }^{[16]}$ Furthermore, healthcare providers working in palliative care settings experience occupational stressors resulting in burnout and other psychosocial problems. A systematic review of eight cross-sectional studies comprised of 1406 health professionals found a prevalence of burnout of $17.3 \%$ among the health professionals. ${ }^{[17]}$ This review also revealed that palliative care nurses had higher emotional exhaustion (19.5\%) and depersonalization (8.2\%), whereas palliative care physicians had lower levels of personal accomplishment (41.2\%). Moreover, $27 \%$ of the social workers who served palliative care patients experienced burnout. These studies indicate a high burden of psychosocial problems among palliative care patients, caregivers, and healthcare providers. Also, the available evidence informs the importance of tackling this enormous psychosocial burden through evidencebased approaches. 


\section{The rise of digital health: Psychosocial interventions in palliative care}

In recent years, a wide range of digital health interventions is being used to deliver psychosocial support, enhance patient-provider communication, and improve health outcomes using a patientcentric approach. Such interventions may bridge the existing gaps of psychosocial care among individuals involved in palliative care processes. For the patients, informal caregivers, and healthcare providers, digitalization of existing services or integration of psychosocial care can play a powerful role, particularly through communication interventions that can support pain management and distress relieve as an important part of palliative care. ${ }^{[18]}$ Such efforts may have a focus on the strategic approach to alleviate the experience of pain, which is often mediated through personal meaning creation processes. Psychosocial interventions promote understanding and control of pain symbolization in palliative care patients that can help with chronic and longterm problems with pain. ${ }^{[19]}$ Most of these interventions are designed to help the sufferers increase their understanding of the causes, patterns, and to mobilize support from healthcare providers and informal caregivers. Digital communication tools, including wearable devices and messaging platforms, may enable to access and share information, and facilitate evidence-based decisionmaking in palliative care.

Digital health technologies are increasingly used in delivering psychosocial support to palliative care patients, as noted in a growing body of scientific literature. A randomized controlled trial assessed the effectiveness of an online support system named "Comprehensive Health Enhancement Support System (CHESS)" in relieving symptom distress among 285 dyads of patients with non-small cell lung cancer (NSCLC) and their primary caregivers for up to 25 months. ${ }^{[20]}$ This trial reported that the CHESS intervention was significantly effective in reducing physical symptom distress at 4 months $(\mathrm{P}=.031$; Cohen $\mathrm{d}=.42)$ and at 6 months $(\mathrm{P}=.004 ; \mathrm{d}=$ 
.61) highlighting that digital interventions can be useful in palliative care populations. Moreover, electronic health applications are increasingly used in palliative care settings globally. Studies by Kallen et al. and Lind et al. reported that digital interventions improved the quality of care and facilitated communication between palliative care patients and providers, ${ }^{[21,22]}$ thus contributing to reduced psychosocial stress that is commonly reported by the patients. From a holistic perspective, palliative care patients and caregivers experience psychosocial challenges while receiving palliative care services, which can be resolved through digital support systems. For example, Maudlin et al. and Pare et al. reported that digital tools can potentially reduce the number of hospitalizations, documentations errors, hospital bed occupancy, and provide more time for direct care, which may alleviate stress among the patients and informal caregivers, leading to better health services outcomes. ${ }^{[23,24]}$

Digital interventions may not only target palliative care problems; rather, they may provide additional support related to health access, information, or mental health resources. ${ }^{[25,26]}$ The adoption of such digital support systems can improve the overall patient experience and satisfaction regarding palliative care. Moreover, such platforms may enable healthcare providers to make real-time decisions for their patients and address occupational concerns and stresses that they may experience. Overall, digital tools can be integrated into institutional or health systems interventions where multiple individuals, including patients, caregivers, and healthcare providers, can interact, emphasizing on how those systems can inform the unmet needs among the individuals and facilitate effective caregiving for everyone. ${ }^{[25]}$ The level and contents of those services can be customized for different groups based on their unique psychosocial needs. For example, mental health components can be incorporated for patients and caregivers who may experience psychological distress or other mental health problems. In contrast, interventions for a social 
worker or healthcare provider may consist of psychosocial support, preventing burnout or empowering those providers to better manage their workload that may contribute to their psychological and emotional wellbeing. Also, digitalization may offer unique opportunities to bridge multiple components, engaging decision-makers, service providers, and users of digital services at different levels. The broader goals of such interventions may focus on optimizing psychosocial outcomes among key stakeholders, whereas specific aims may emphasize teambased outcomes, institutional performances, or systems-level outcomes in palliative care. ${ }^{[21-23,25]}$ An integrated approach can better inform how the health system and its components are working together to address psychosocial challenges that may impact lives engaged at different phases of palliative care.

\section{Implementing digital interventions in palliative care: critical challenges and recommendations}

Healthcare decision-makers need to acknowledge critical issues that may affect the implementation of psychosocial interventions in the digital era and address the same through evidence-based measures. One of the major challenges associated with the development of digital interventions is the unique psychosocial needs of the potential users of those services. ${ }^{[27,28]}$ For example, the levels and contents of psychosocial support for the patients, caregivers, and healthcare providers can be different necessitating customization and optimization of any such interventions. Moreover, some interventions may involve dyads or groups that may facilitate the sharing of information and work together to manage physical and psychosocial problems. ${ }^{[25]}$ One recommendation that can be adopted is to analyze the complex needs of the primary users of digital interventions so that those issues can be addressed in a planned way. 
The existing literature on psychosocial interventions leveraging digital technologies shows a growing number of studies from high-income countries. ${ }^{[29-31]}$ As low- and middle-income countries (LMICs) have a higher proportion of palliative care needs and associated psychosocial challenges, ${ }^{[32]}$ their under-representation in the scientific evidence shows a critical gap of implementation research in those countries. This lack of evidence may be a result of inadequate digital interventions or integration of digital health in palliative care in those contexts. This would require active efforts to promote context-specific research on how emerging digital tools can be used for psychosocial care alongside palliative care delivery. Such interventions should be developed considering the unique needs of the patients, informal caregivers, and palliative care providers.

Another major issue is the socioeconomic divide in different contexts. Even in high-income countries, all population groups may not have similar healthcare or digital services. People with better internet access, household wealth, insurance, and socioeconomic advantaged may have higher access to digital health services. ${ }^{[33]}$ This highlights that the availability of digital technologies or palliative care in a country may not ensure all people in need can access the same. Moreover, people who are homeless or forcibly displaced may have a higher burden of psychosocial problems. ${ }^{[34,35]}$ Delivering palliative care and associated psychosocial support to those marginalized people can be challenging. ${ }^{[36]}$ Extensive research is needed to explore such intersections of health challenges that may guide the development of future palliative care overcoming socioeconomic disparities across populations. It is critical to explore the population health challenges and examine how those may impact the use of digital psychosocial interventions in palliative care settings. Healthcare organizations and health systems should play a proactive role in addressing such complex multilevel challenges to deliver digital support to palliative care 
patients and caregivers who may not have the ability to access the same. Designing publicly funded interventions, mobilizing social and community resources, and engaging philanthropic and nonprofit institutions to maximize the usage and coverage of those services may help in this regard.

Resilience is a persistent challenge in delivering health services, especially in public health emergencies, when most service networks are disrupted. For instance, during the coronavirus disease (COVID-19) pandemic, the patients, caregivers, and healthcare providers may have experienced unique psychosocial stressors resulting in adverse mental health outcomes. ${ }^{[37-41]}$ Such public health emergencies may impact palliative care services and may contribute to additional psychosocial challenges for people involved in palliative care processes. ${ }^{[26]}$ As digitalization offers teleconsultations and remote caregiving, future digital interventions should be developed considering social and environmental challenges that may affect or alter palliative care services. It is necessary to synthesize the lessons learned during the COVID-19 pandemic to build resilient palliative care leveraging technological advancements and improving psychosocial health outcomes.

The adoption of and adherence to psychosocial interventions may need closer attention to the complex sociocultural issues such as stigma to mental health issues and cultural appropriateness of non-pharmacological therapies. ${ }^{[42]}$ Moreover, the pattern of technology use and other dimensions of digital health behavior may play critical roles in this regard. It is necessary to assess the individual and sociocultural issues that may inform the development of digital interventions and affect the adoption of the same. As the current evidence base on socio-behavioral aspects of digital health in palliative care is limited, more research is needed in this emerging domain to better understand the factors that may influence psychosocial health outcomes among individuals and populations. ${ }^{[27]}$ Also, engaging mental and behavioral scientists and practitioners in planning and 
implementing digital services for palliative care can provide expert inputs that may address major concerns in different populations.

Capacity building is another major challenge for implementing digital health interventions globally. ${ }^{[30,43,44]}$ Healthcare providers and other stakeholders who may contribute to the effective delivery of digital health services may require educational interventions for meaningful participation in digital care systems. It would be critical to incorporate medical and psychosocial care in existing systems, whereas newer systems may have layouts necessitating a brief orientation to the potential users. In addition, information technology providers engaged in digital health services should be introduced to healthcare issues that would be relevant for troubleshooting the digital services in palliative care settings. In this context, healthcare providers may leverage advanced technologies such as artificial intelligence or geospatial analyses that are increasingly used for improving health outcomes in vulnerable populations. ${ }^{[43,45,46]}$ Nonetheless, the evolution of digital health technologies and the changing landscape of palliative care systems would need a continuous, evidence-guided, and multipronged approach that aims to improve individual and institutional capacities for offering digital health services in palliative care.

Digitalizing palliative care and improving psychosocial health outcomes would need proactive efforts for strengthening health systems. Palliative care is still under-developed in most nations globally. This challenge is more evident in LMICs, where pluralistic health systems may not perform optimally to prevent and treat chronic diseases. ${ }^{[32]}$ Also, most people in those contexts may not have equitable access to palliative care that might be available in a limited way. Universal health coverage is often envisaged to reach the marginalized population, but specialized services such as palliative care may not be readily available for everyone. This unmet need for palliative care may not be addressed through isolated efforts taken by individual providers or localized 
organizations. Scholars and practitioners may explore how health problems requiring palliative care services can be prevented so that palliative care providers have a reduced workload, and more resources can be allocated to provide psychosocial care to the affected individuals. Moreover, digitalizing psychosocial services in palliative care should be consistent with the societal goals of digitalizing health systems and other welfare systems. Effective healthcare leadership and stewardship approaches would be necessary to make lasting commitments to prevent psychosocial problems and improve palliative care across populations.

\section{Conclusions}

Psychosocial challenges continue to impact health and wellbeing among palliative care patients, informal caregivers, and healthcare providers. Despite a growing body of evidence on psychosocial burden across palliative care populations, little efforts are made to address such problems. Evidence-based digital interventions are being used for improving healthcare services and multiple health outcomes in palliative care settings. Critical challenges may affect the widespread adoption of such interventions, especially in low-resource contexts. Future research should explore individual and population-based barriers and facilitators, whereas health services research is needed to better inform how healthcare providers and organizations can contribute to such developments. Lastly, health disparities should be addressed through health systems interventions that may empower key decision-makers and organizations to adopt digital services and alleviate the psychosocial burden in palliative care populations. 
Conflicts of interest: None

Funding: No funding was received for conceptualizing or preparing this manuscript.

\section{References}

1. Bajwah S, Higginson IJ, Ross JR, Wells AU, Birring SS, Riley J, et al. The palliative care needs for fibrotic interstitial lung disease: A qualitative study of patients, informal caregivers and health professionals. Palliat Med. 2013; 27(9):869-76. Available from: http://journals.sagepub.com/doi/10.1177/0269216313497226

2. Jansen L, Dauphin S, van den Akker M, De Burghgraeve T, Schoenmakers B, Buntinx F. Prevalence and predictors of psychosocial problems in informal caregivers of older cancer survivors - A systematic review: Still major gaps in current research. Eur J Cancer Care (Engl) 2018; 27(6):e12899. Available from: http://doi.wiley.com/10.1111/ecc.12899

3. Vachon MLS, Kristjanson L, Higginson I. Psychosocial issues in palliative care: The patient, the family, and the process and outcome of care. J Pain Symptom Manage 1995;10(2):142-50.

4. Solway E, Estes CL, Goldberg S, Berry J. Access Barriers to Mental Health Services for Older Adults from Diverse Populations: Perspectives of Leaders in Mental Health and Aging. J Aging Soc Policy. 2010; 22(4):360-78. Available from: http://www.tandfonline.com/doi/abs/10.1080/08959420.2010.507650

5. McCabe MP, Leas L. A qualitative study of primary health care access, barriers and satisfaction among people with mental illness. Psychol Health Med. 2008; 13(3):303-12. Available from: 
http://www.tandfonline.com/doi/abs/10.1080/13548500701473952

6. Hossain MM, Hasan MT, Sultana A, Faizah F. New Mental Health Act in Bangladesh: unfinished agendas. The Lancet Psychiatry. 2019; 6(1):e1. DOI: 10.1016/S2215-0366(18)30472-3

7. Hossain M, Purohit N. Improving child and adolescent mental health in India: Status, services, policies, and way forward. Indian J. Psychiatry. 2019;61(4):415-9. Available from: https://www.ncbi.nlm.nih.gov/pmc/articles/PMC6657557/, DOI: 10.4103/psychiatry.IndianJPsychiatry_217_18

8. Hossain MM. Health and well-being of cancer caregivers in a changed role of breadwinners. Indian J. Cancer. 2018;55(4):422. Available from: https://pubmed.ncbi.nlm.nih.gov/30829286/, DOI: 10.4103/ijc.IJC_266_18

9. Lynch T, Connor S, Clark D. Mapping levels of palliative care development: A global update. J Pain Symptom Manage 2013;45(6):1094-106.

10. Dueñas M, Ojeda B, Salazar A, Mico JA, Failde I. A review of chronic pain impact on patients, their social environment and the health care system. J. Pain Res. 2016; 9:457-67. Available from: /pmc/articles/PMC4935027/?report=abstract

11. Koh MYH, Chong PH, Neo PSH, Ong YJ, Yong WC, Ong WY, et al. Burnout, psychological morbidity and use of coping mechanisms among palliative care practitioners: A multi-centre crosssectional study. Palliat Med . 2015;29(7):633-42. Available from: http://journals.sagepub.com/doi/10.1177/0269216315575850

12. Slocum-Gori S, Hemsworth D, Chan WWY, Carson A, Kazanjian A. Understanding Compassion Satisfaction, Compassion Fatigue and Burnout: A survey of the hospice palliative care workforce. Palliat. Med. 2013;27(2):172-8. Available from: http://journals.sagepub.com/doi/10.1177/0269216311431311 
13. Wilson KG, Chochinov HM, Graham Skirko M, Allard P, Chary S, Gagnon PR, et al. Depression and Anxiety Disorders in Palliative Cancer Care. J Pain Symptom Manage 2007;33(2):118-29.

14. O'Connor M, White K, Kristjanson LJ, Cousins K, Wilkes L. The prevalence of anxiety and depression in palliative care patients with cancer in Western Australia and New South Wales. Med J Aust 2010;193(S5):S44-7. Available from: https://onlinelibrary.wiley.com/doi/abs/10.5694/j.1326-5377.2010.tb03927.x

15. Mitchell AJ, Chan M, Bhatti H, Halton M, Grassi L, Johansen C, et al. Prevalence of depression, anxiety, and adjustment disorder in oncological, haematological, and palliative-care settings: A meta-analysis of 94 interview-based studies. Lancet Oncol 2011;12(2):160-74.

16. Adol EA. Prevalence of depression among caregivers of patients receiving palliative care in Kenyatta national hospital. UoN. 2014;Available from: http://erepository.uonbi.ac.ke/handle/11295/76670

17. Parola V, Coelho A, Cardoso D, Sandgren A, Apóstolo J. Prevalence of burnout in health professionals working in palliative care. JBI Database Syst Rev Implement Reports. 2017;15(7):1905-33. Available from: http://journals.lww.com/01938924-201707000-00015

18. Kreps G. Communication and Palliative Care: E-Health Interventions and Pain Management. In: Handbook of Pain and Palliative Care. Springer International Publishing; 2018. page 7181.Available from: https://link.springer.com/chapter/10.1007/978-3-319-95369-4_5

19. Cherny NI, Coyle N, Foley KM. The treatment of suffering when patients request elective death.. J. Palliat. Care. 1994;10(2):71-9. Available from: http://journals.sagepub.com/doi/10.1177/082585979401000212

20. Gustafson DH, DuBenske LL, Namkoong K, Hawkins R, Chih M-Y, Atwood AK, et al. An eHealth system supporting palliative care for patients with non-small cell lung cancer. Cancer 
2013;119(9):1744-51. Available from: http://doi.wiley.com/10.1002/cncr.27939

21. Kallen MA, Yang DS, Haas N. A technical solution to improving palliative and hospice care. Support Care Cancer. 2012;20(1):167-74. Available from: https://pubmed.ncbi.nlm.nih.gov/21240650/

22. Lind L, Karlsson D, Fridlund B. Digital pens and pain diaries in palliative home health care: Professional caregivers' experiences. Med Inform Internet Med 2007;32(4):287-96.

23. Maudlin J, Keene J, Kobb R. A road map for the last journey: Home telehealth for holistic end-oflife care. Am. J. Hosp. Palliat. Med. 2006;23(5):399-403. Available from: https://pubmed.ncbi.nlm.nih.gov/17060308/

24. Paré G, Sicotte C, Chekli M, Jaana M, De Blois C, Bouchard M. A pre-post evaluation of a telehomecare program in oncology and palliative care. Telemed e-Health. 2009;15(2):154-9. Available from: https://pubmed.ncbi.nlm.nih.gov/19292624/

25. Hong YA, Hossain MM, Chou WS. Digital interventions to facilitate patient-provider communication in cancer care: A systematic review. Psychooncology. 2020;29(4):591-603. Available from: https://onlinelibrary.wiley.com/doi/abs/10.1002/pon.5310, DOI: 10.1002/pon.5310

26. Lai L, Sato R, Ouchi K, Landman AB, Zhang HM. Digital health innovation to integrate palliative care during the COVID-19 pandemic. Am J Emerg Med. 2020;0(0). Available from: https://doi.org/10.1016/j.ajem.2020.08.008

27. Demiris G, Parker Oliver D, Wittenberg-Lyles E. Technologies to support end-of-life care. Semin Oncol Nurs. 2011;27(3):211-7. Available from: /pmc/articles/PMC3143374/?report=abstract

28. Hassankhani H, Rahmani A, Best A, Taleghani F, Sanaat Z, Dehghannezhad J. Barriers to homebased palliative care in people with cancer: A qualitative study of the perspective of caregivers. 
Nurs Open. 2020;7(4):1260-8. Available from:

https://onlinelibrary.wiley.com/doi/abs/10.1002/nop2.503

29. Tasnim S, Trisha NF, Fan Q, Sultana A, Mishu SH, Hasan NT, et al. Improving mental health and wellbeing among sexual and gender minorities using digital interventions: a systematic review. PsyArXiv. 2020;Available from: https://psyarxiv.com/9wu42/, DOI: 10.31234/osf.io/9wu42

30. Hossain MM, Tasnim S, Sharma R, Sultana A, Shaik AF, Faizah F, et al. Digital interventions for people living with non-communicable diseases in India: A systematic review of intervention studies and recommendations for future research and development. Digital Health. 2019; 5:205520761989615. Available from: http://journals.sagepub.com/doi/10.1177/2055207619896153, DOI: 10.1177/2055207619896153

31. Gibson DG, Tamrat T, Mehl G. The state of digital interventions for demand generation in lowand middle-income countries: Considerations, emerging approaches, and research gaps. Glob Heal Sci Pract. 2018;6(Suppl 1):S49-60. Available from:/pmc/articles/PMC6203418/?report=abstract

32. Poudel A, Bhuvan KC, Shrestha S, Nissen L. Access to palliative care: Discrepancy among lowincome and high-income countries. J Glob Health. 2019;9(2):20309. Available from: https://www.ncbi.nlm.nih.gov/pmc/articles/PMC6812938/

33. Graetz I, Huang J, Brand RJ, Hsu J, Yamin CK, Reed ME. Bridging the Digital Divide: Mobile Access to Personal Health Records Among Patients With Diabetes. Am J Manag Care. 2018;24(1):43-8. Available from: www.ajmc.com

34. Hossain MM, Purohit N. Protecting Rohingya: lives, minds, and the future. Lancet. 2018; 391(10120):533. DOI: https://doi.org/10.1016/S0140-6736(18)30209-5

35. Hossain MM, Sultana A, Tasnim S, Fan Q, Ma P, McKyer ELJ, et al. Prevalence of mental disorders among people who are homeless: An umbrella review. Int. J. Soc. Psychiatry. 
2020;66(6):528-41. Available from: http://journals.sagepub.com/doi/10.1177/0020764020924689, DOI: https://doi.org/10.1177/0020764020924689

36. Collier R. Bringing palliative care to the homeless. CMAJ. 2011;183(6):E317-8. Available from: www.cmaj.ca/cgi/doi/10.1503/cmaj.109-3793

37. Chi X, Becker B, Yu Q, Willeit P, Jiao C, Huang L, et al. Prevalence and Psychosocial Correlates of Mental Health Outcomes Among Chinese College Students During the Coronavirus Disease (COVID-19) Pandemic. Front Psychiatry. 2020;11:1. Available from:

https://www.frontiersin.org/article/10.3389/fpsyt.2020.00803/full

38. Hossain M, Tasnim S, Faizah F, Sultana A, Mazumder H, Zou L, et al. Epidemiology of mental health problems in COVID-19: a review , PsyArXiv. 2020; Available from: https://psyarxiv.com/q8e5u/, DOI: 10.31234/osf.io/q8e5u

39. Sultana A, Sharma R, Hossain MM, Bhattacharya S, Purohit N. Burnout Among Healthcare Providers During COVID-19 Pandemic: Challenges and Evidence-based Interventions. Indian J Med Ethics. 2020;Available from: https://osf.io/preprints/socarxiv/4hxga/, DOI: 10.20529/IJME.2020.73

40. Mazumder H, Hossain MM, Das A. Geriatric Care during Public Health Emergencies: Lessons Learned from Novel Corona Virus Disease (COVID-19) Pandemic. J Gerontol Soc Work. 2020;63(4):257-8. Available from: https://www.tandfonline.com/doi/abs/10.1080/01634372.2020.1746723, DOI: $10.1080 / 01634372.2020 .1746723$

41. Sharma R, Hossain MM, Pawar P, Sharma S. Air pollution and geriatric mental health: Perspectives on the COVID-19 pandemic. Int. Psychogeriatrics. 2020;1-2. Available from: https://doi.org/10.1017/S1041610220001428 
42. Fung KMT, Tsang HWH, Corrigan PW. Self-stigma of people with schizophrenia as predictor of their adherence to psychosocial treatment. Psychiatr Rehabil J 2008;32(2):95-104.

43. Hossain MM, Sharma R, Sultana A, Tasnim S, Faizah F. Globalising artificial intelligence for improved clinical practice. Indian J Med Ethics. 2019;01-2. Available from: https://europepmc.org/article/med/31865285, DOI: 10.20529/IJME.2019.074

44. Curioso WH. Building capacity and training for digital health: Challenges and opportunities in Latin America. J. Med. Internet Res. 2019;21(12):e16513. Available from: https://www.jmir.org/2019/12/e16513/

45. Ahasan R, Alam MS, Chakraborty T, Hossain MM. Applications of GIS and Geospatial analyses in COVID-19 research: A systematic review. SocArXiv. 2020;Available from: https://osf.io/preprints/socarxiv/zqmwa/, DOI: 10.31235/osf.io/zqmwa

46. Ahasan R, Hossain MM. Leveraging GIS Technologies for Informed Decision-making in COVID19 Pandemic. SocArXiv. 2020; Available from: https://osf.io/preprints/socarxiv/v6nuf/, DOI: 10.31235/osf.io/v6nuf 